\title{
Successful Treatment of a Neonate with Idiopathic Persistent Pulmonary Hypertension with Inhaled Nitric Oxide via Nasal Cannula without Mechanical Ventilation
}

\author{
Jayasree Nair, M.D. ${ }^{1}$ Joseph Orie, M.D. ${ }^{2}$ Satyan Lakshminrusimha, M.D. ${ }^{1}$ \\ ${ }^{1}$ Division of Neonatology, Women and Children's Hospital of Buffalo, \\ State University of New York at Buffalo, Buffalo, New York \\ 2 Pediatric Cardiology Associates, Buffalo, New York

\begin{abstract}
Address for correspondence and reprint requests Jayasree Nair, M.D., Division of Neonatology, Women and Children's Hospital of Buffalo, 219 Bryant St, Buffalo, NY 14222; State University of New York at Buffalo, Buffalo, NY (e-mail: nairjayasree@yahoo.com).
\end{abstract}

\begin{abstract}
Keywords

- hypoxemic respiratory failure

- noninvasive ventilation

We report a case study of a term neonate presenting with oxygen desaturation without respiratory distress or acidosis, despite receiving $100 \%$ oxygen through a nasal cannula. Echocardiogram showed evidence of persistent pulmonary hypertension of the newborn (PPHN). She was successfully treated with inhaled nitric oxide (iNO) via nasal cannula without requiring mechanical ventilation. In a term neonate with idiopathic PPHN with adequate respiratory drive without any parenchymal lung disease, noninvasive methods of iNO delivery may treat the condition without the complications associated with mechanical ventilation.
\end{abstract}

Persistent pulmonary hypertension of the newborn (PPHN) is a syndrome of failed circulatory adaptation at birth, characterized by high pulmonary vascular resistance causing extrapulmonary left-to-right shunting of blood across the ductus arteriosus and/or foramen ovale. ${ }^{1}$ Selective pulmonary vasodilatation with inhaled nitric oxide (iNO) has been proven to improve oxygenation in term newborns with severe hypoxemic respiratory failure (HRF) and PPHN. ${ }^{2,3}$ Randomized controlled trials have demonstrated that iNO significantly decreased the need for extracorporeal membrane oxygenation in newborns greater than 34 weeks' gestational age with $\mathrm{HRF}^{4,5}$ Traditionally, iNO has been delivered to intubated and mechanically ventilated babies when they continue to be hypoxemic despite administration of high levels of inspired oxygen. Infants with PPHN suffer from long-term morbidity such as chronic lung disease, neurological morbidity, and sensorineural deafness. ${ }^{6}$ Some of these long-term consequences may be secondary to mechanical ventilation. The use of iNO administered by a noninvasive method like nasal cannula has been only anecdotally described, usually during the weaning process after the neonate has been mechanically ventilated. ${ }^{7}$ We report a case of idiopathic PPHN treated successfully with iNO delivered through a nasal cannula, avoiding mechanical ventilation.

\section{Case Report}

A female infant weighing $2994 \mathrm{~g}$ was born at 39 weeks' gestation by spontaneous vaginal delivery to a 16 -year-old primigravida. Her antenatal history was unremarkable. There was no history of any medication intake and no history of infections. There were no inherited disorders reported in the family and no history of alcohol, drugs, or cigarette smoking. The baby was born after rupture of membranes for 6 hours through meconium-stained amniotic fluid with Apgar scores of 9 and 9 at 1 and 5 minutes, respectively. She was vigorous at birth, received routine care at delivery, and was transferred to the well baby nursery.

She remained asymptomatic for the next 36 hours, when during a feed she was noted to be gray and tachypneic with a respiratory rate of $70 / \mathrm{min}$. Her oxygen saturations were received

August 26, 2011

accepted after revision

November 18, 2011

published online

February 22, 2012
Copyright $\odot 2012$ by Thieme Medical Publishers, Inc., 333 Seventh Avenue, New York, NY 10001, USA. Tel: +1(212) 584-4662.
DOI http://dx.doi.org/ 10.1055/s-0032-1305797. ISSN 2157-6998. 


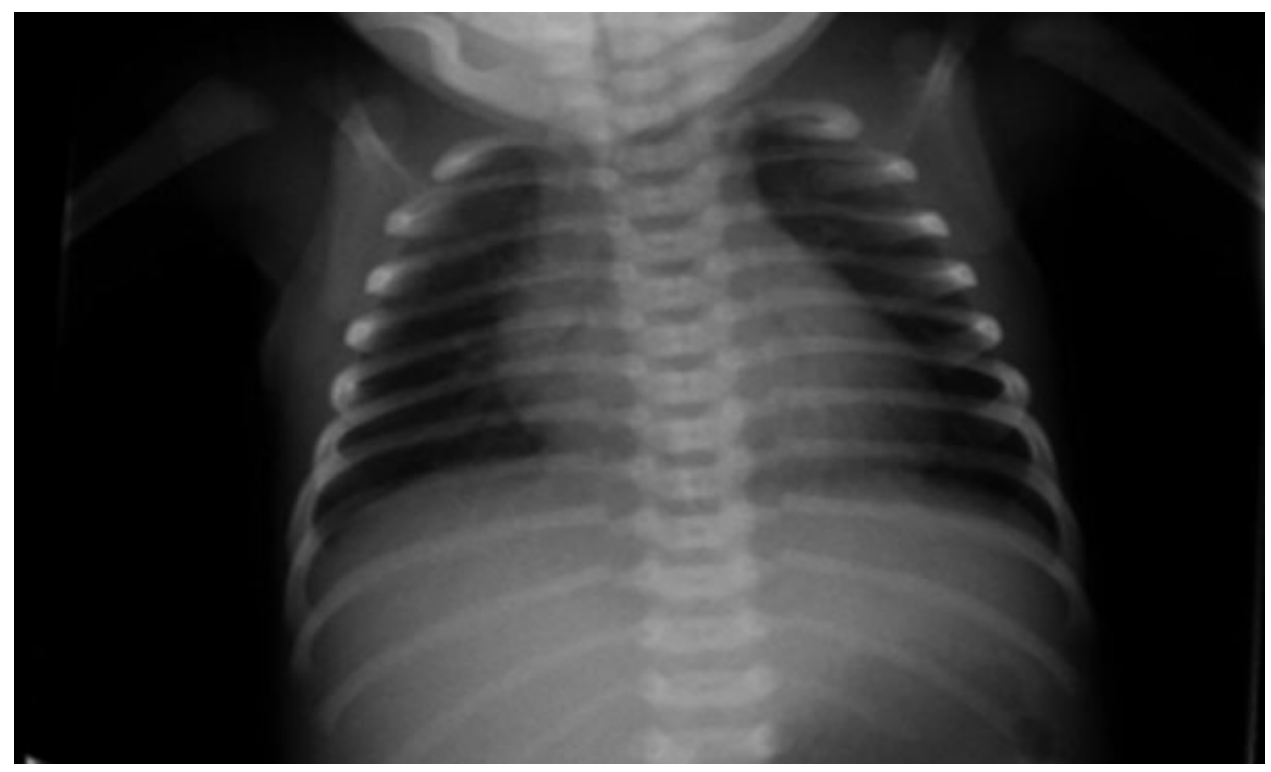

Figure 1 Chest radiograph showing clear lung fields without any evidence of parenchymal lung disease suggestive of idiopathic persistent pulmonary hypertension of the newborn or "black lung disease."

noted to be 79 to $80 \%$ with a loud systolic murmur heard over the left heart border, which had not been previously documented. She had an arterial blood gas with a $\mathrm{pH}$ of $7.38, \mathrm{PacO}_{2}$ of $44 \mathrm{~mm} \mathrm{Hg}$ and a partial pressure of oxygen $\left(\mathrm{Po}_{2}\right)$ of $47 \mathrm{~mm}$ $\mathrm{Hg}$ on $40 \%$ oxygen with an A-a gradient of $182 \mathrm{~mm} \mathrm{Hg}$. A hyperoxia test in $100 \%$ oxygen raised her $\mathrm{Po}_{2}$ to $372 \mathrm{~mm} \mathrm{Hg}$.

A chest X-ray showed clear lung fields with no significant abnormality (-Fig.1). An echocardiogram revealed moderate tricuspid regurgitation (estimated right ventricular systolic pressure $\sim 90 \mathrm{~mm} \mathrm{Hg}$; - Fig. 2). Pulmonary venous return appeared normal to the left atrium without any evidence of obstruction. The ductus arteriosus was not visualized. The baby was maintaining oxygen saturations of $90 \%$ in room air, but was tachypneic with respiratory rates of 70 to $80 / \mathrm{min}$. Intermittent periods of desaturation were observed requiring escalation of inspired oxygen concentration to 100\%. Oxygen saturations continued to drop below $90 \%$ periodically, despite receiving $2 \mathrm{~L} / \mathrm{min}$ of $100 \%$ oxygen by nasal cannula. She was transferred to the neonatal intensive care unit for further management.

In the intensive care unit, umbilical arterial and venous lines were placed for serial blood gas and central venous pressure monitoring. iNO was started at $20 \mathrm{ppm}$ through a nasal cannula with $2 \mathrm{~L} / \mathrm{min}$ flow. The inspired oxygen was weaned down to $40 \%$ after initiation of iNO. Her first blood gas after iNO was started revealed a $\mathrm{pH}$ of 7.39 with a $\mathrm{Po}_{2}$ of $68 \mathrm{~mm} \mathrm{Hg}$ and a $\mathrm{pCO}_{2}$ of $44 \mathrm{~mm} \mathrm{Hg}$ on $40 \%$ oxygen by nasal cannula. Episodes of oxygen desaturation were not observed during therapy with iNO.

Repeat echocardiogram after 24 hours of iNO therapy did not reveal tricuspid regurgitation. Thereafter, iNO was weaned by 5 ppm every 4 hours per unit protocol for PPHN. After the first wean from 20 to 15 ppm, the infant experienced some tachypnea and desaturation, for which she was placed back on iNO 20 ppm for another 24 hours.
Following this period, she tolerated the iNO wean better and was off nitric oxide by day 4 of life. Repeat echocardiogram done prior to discharge and after being off iNO for 24 hours showed trace tricuspid regurgitation (estimated right ventricular systolic pressure 25 to $30 \mathrm{~mm} \mathrm{Hg}$ ). No patent ductus arteriosus was visualized, and there was good left ventricular function. She was discharged home with close cardiology follow-up. Repeat echocardiogram 1 month after discharge revealed no tricuspid regurgitation and no PDA, with normal cardiac function. The baby herself was asymptomatic in room air and growing well.

The etiology of pulmonary hypertension resulting in tricuspid regurgitation in this baby was not clear but could be secondary to antenatal ductal closure resulting in PPHN from vascular remodeling. ${ }^{8}$

\section{Discussion}

Barotrauma and volutrauma are often associated with prolonged mechanical ventilation in newborn infants. ${ }^{9,10}$ In this case report, we describe an infant with idiopathic PPHN successfully treated with iNO, avoiding the hazards of mechanical ventilation. To our knowledge, this is the first report of using iNO by nasal cannula as primary therapy for PPHN in the immediate newborn period. Noninvasive methods of delivering iNO have been anecdotally described in the literature. The fact that iNO can be delivered effectively by continuous flow nasal cannulae was first demonstrated in a young infant with primary pulmonary hypertension, who was treated with nasal nitric oxide for several weeks until approved for enrollment into the long-term intravenous prostacyclin therapy program. ${ }^{11}$ iNO via a nasopharyngeal tube was used in a 145-day-old infant with a severely hypoplastic lung and end-stage pulmonary hypertension, in whom clinical improvement was maintained for 7 days. ${ }^{12}$ Use of pulsed 

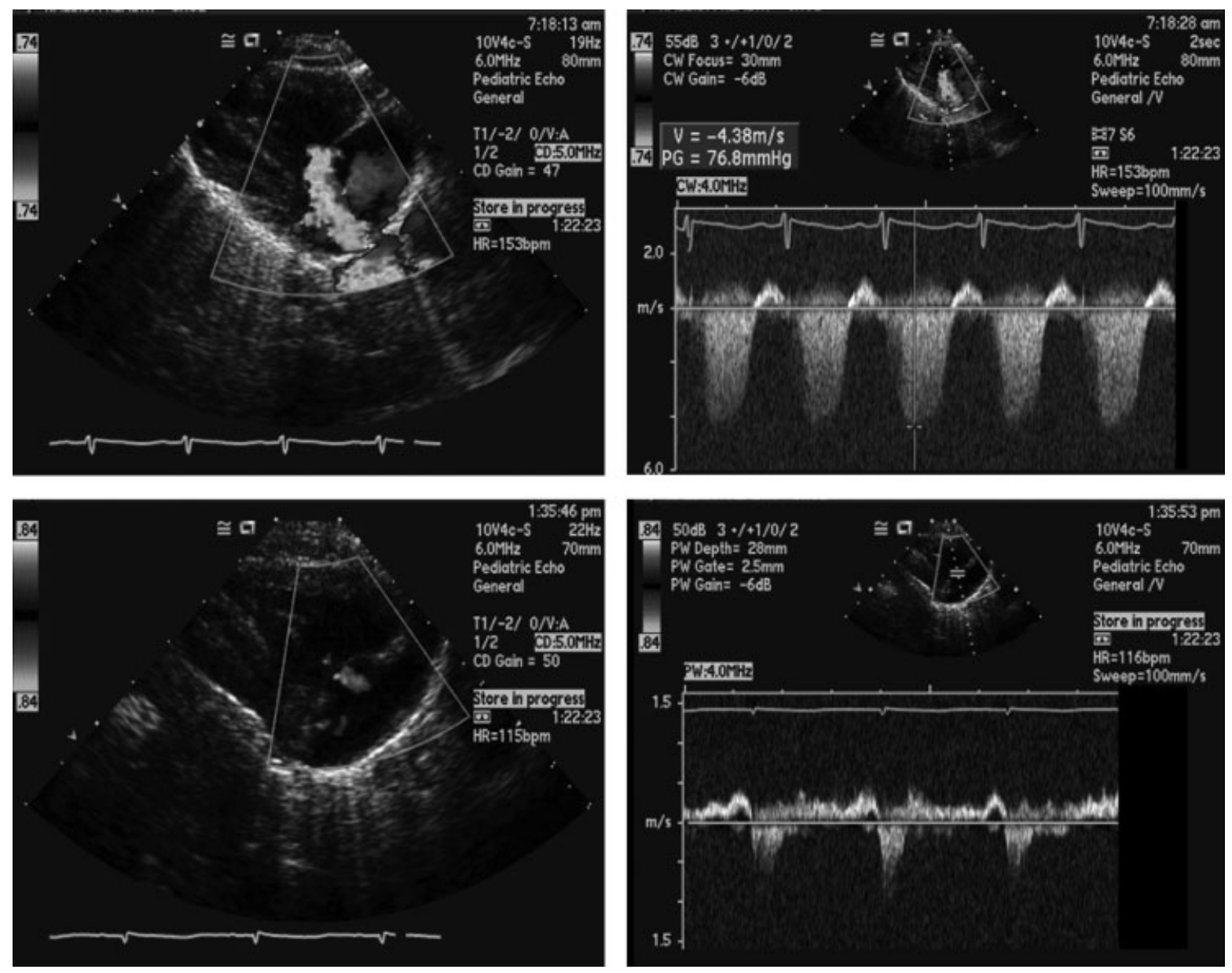

Figure 2 Echocardiography revealing a tricuspid regurgitation jet indicative of moderate pulmonary hypertension in the upper frames. On inhaled nitric oxide, the right ventricular pressures are seen to decrease in the lower frames.

nasal delivery of nitric oxide was described in adults with primary pulmonary hypertension in the home setting. ${ }^{13}$ Premature infants received iNO therapy through continuous positive airway pressure and nasal cannula in the weaning phase in the Nitric Oxide to Prevent Chronic Lung Disease (NO-CLD) trial for prevention of bronchopulmonary dysplasia without any negative consequences. ${ }^{14}$ Noninvasive delivery of iNO therapy has also been reported for late pulmonary hypertension in newborn infants with congenital diaphragmatic hernia. In a case series, patients who had suprasystemic pulmonary hypertension when iNO was discontinued before extubation were treated with iNO through a nasal cannula. In the same study, nasopharyngeal nitric oxide concentrations were $5.4 \pm 0.5 \mathrm{ppm}$ and $2.4 \pm 0.4 \mathrm{ppm}$ with iNO measured proximally in the delivery device at 10 and $5 \mathrm{ppm}$, respectively. ${ }^{7}$ In another case series, the tracheal concentration of nitric oxide was measured using a chemiluminescence device during pulsed nasal nitric oxide delivery at 20,40 , and $60 \mathrm{~mL} /$ breath and found to range between 28 and 38 ppm. ${ }^{15}$

The feasibility of nitric oxide administration by oxygen hood in neonatal pulmonary hypertension has been studied in piglets. ${ }^{16}$ It was found that nitric oxide decreased $(>50 \%)$ pulmonary artery pressure and vascular resistance in both hypoxia- and Group B streptococcus-induced pulmonary hypertension, with minimal effects on systemic arterial pressure and cardiac output.

A masked randomized controlled pilot trial using iNO via oxygen hood was conducted in $>34$-weeks gestational age infants with high A-a gradient ( 400 to $600 \mathrm{~mm} \mathrm{Hg}$ ) with the primary outcome measure being partial pressure of oxygen in the alveoli an hour after starting study gas. This study did not complete enrollment but the authors concluded that administration of iNO by oxygen hood is feasible, but required larger controlled trials. ${ }^{17}$

Our patient had symptomatic pulmonary hypertension as demonstrated by her labile hypoxemia. Echocardiogram supported this diagnosis and ruled out structural heart disease. With adequate respiratory drive, no evidence of parenchymal lung disease on the chest radiograph, and no signs of respiratory distress except for mild tachypnea, we felt she did not warrant intubation and mechanical ventilation, which is associated with chronic lung injury. iNO was delivered through the INOmax DS (Ikaria Inc., Hampton, NJ) with the circuit as shown in the product manual. Though we did not measure environmental nitric oxide levels, previous studies have shown negligible leakage of iNO with noninvasive methods of delivery. $12,16,17$ 
We conclude that in newborn infants presenting with hypoxic repiratory failure with hypoxemia without hypercarbia, respiratory distress, or evidence of parenchymal lung disease, therapy with iNO using a nasal cannula may reduce exposure to high levels of inspired oxygen and avoid mechanical ventilation. Randomized and double blinded trials evaluating this therapeutic modality are warranted.

\section{Disclosures}

Dr. Lakshminrusimha is a member of the speaker's bureau for Ikaria. There are no other relevant financial relationships to disclose.

\section{References}

1 Konduri GG, Kim UO. Advances in the diagnosis and management of persistent pulmonary hypertension of the newborn. Pediatr Clin North Am 2009;56:579-600

2 Roberts JD, Polaner DM, Lang P, Zapol WM. Inhaled nitric oxide in persistent pulmonary hypertension of the newborn. Lancet 1992; 340:818-819

3 Davidson D, Barefield ES, Kattwinkel J, et al; The I-NO/PPHN Study Group. Inhaled nitric oxide for the early treatment of persistent pulmonary hypertension of the term newborn: a randomized, double-masked, placebo-controlled, dose-response, multicenter study. Pediatrics 1998;101(3 Pt 1):325-334

4 The Neonatal Inhaled Nitric Oxide Study Group. Inhaled nitric oxide in full-term and nearly full-term infants with hypoxic respiratory failure. N Engl J Med 1997;336:597-604

5 Clark RH, Kueser TJ, Walker MW, et al; Clinical Inhaled Nitric Oxide Research Group. Low-dose nitric oxide therapy for persistent pulmonary hypertension of the newborn. N Engl J Med 2000; 342:469-474
6 Leavitt AM, Watchko JF, Bennett FC, Folsom RC. Neurodevelopmental outcome following persistent pulmonary hypertension of the neonate. J Perinatol 1987;7:288-291

7 Kinsella JP, Parker TA, Ivy DD, Abman SH. Noninvasive delivery of inhaled nitric oxide therapy for late pulmonary hypertension in newborn infants with congenital diaphragmatic hernia. J Pediatr 2003;142:397-401

8 Alano MA, Ngougmna E, Ostrea EM Jr, Konduri GG. Analysis of nonsteroidal antiinflammatory drugs in meconium and its relation to persistent pulmonary hypertension of the newborn. Pediatrics 2001;107:519-523

9 Hayes D Jr, Feola DJ, Murphy BS, Shook LA, Ballard HO. Pathogenesis of bronchopulmonary dysplasia. Respiration 2010;79:425-436

10 Miller JD, Carlo WA. Pulmonary complications of mechanical ventilation in neonates. Clin Perinatol 2008;35:273-281, x-xi

11 Ivy DD, Wiggins JW, Badesch DB, Kinsella JP, Kelminson LL, Abman SH. Nitric oxide and prostacyclin treatment of an infant with primary pulmonary hypertension. Am J Cardiol 1994;74:414-416

12 Kakuya F, Takase M, Ishii N, et al. Inhaled nitric oxide therapy via nasopharyngeal tube in an infant with end-stage pulmonary hypertension. Acta Paediatr Jpn 1998;40:155-158

13 Channick RN, Newhart JW, Johnson FW, et al. Pulsed delivery of inhaled nitric oxide to patients with primary pulmonary hypertension: an ambulatory delivery system and initial clinical tests. Chest 1996;109:1545-1549

14 Ballard RA, Truog WE, Cnaan A, et al; NO CLD Study Group. Inhaled nitric oxide in preterm infants undergoing mechanical ventilation. N Engl J Med 2006;355:343-353

15 Ivy DD, Parker D, Doran A, Parker D, Kinsella JP, Abman SH. Acute hemodynamic effects and home therapy using a novel pulsed nasal nitric oxide delivery system in children and young adults with pulmonary hypertension. Am J Cardiol 2003;92:886-890

16 Ambalavanan N, St John E, Carlo WA, Bulger A, Philips JB III. Feasibility of nitric oxide administration by oxygen hood in neonatal pulmonary hypertension. J Perinatol 2002;22:50-56

17 Ambalavanan N, El-Ferzli GT, Roane C, Johnson R, Carlo WA. Nitric oxide administration using an oxygen hood: a pilot trial. PLoS ONE 2009;4:e4312 